\title{
Urticaria, CTCAE
}

National Cancer Institute

\section{Source}

National Cancer Institute. Urticaria, CT CAE. NCI Thesaurus. Code C58019.

A disorder characterized by an itchy skin eruption characterized by wheals with pale interiors and well-defined red margins. 